OPEN ACCESS

Edited by:

Tomoharu Sugie,

Kansai Medical University Hospital,

Japan

Reviewed by:

Alba Di Leone,

Università Cattolica del Sacro

Cuore, Italy

Dorin Dumitru,

Hull and East Yorkshire Hospitals

NHS Trust, United Kingdom

*Correspondence:

Masahiro Takada

masahiro@kuhp.kyoto-u.ac.jp

Specialty section:

This article was submitted to

Surgical Oncology,

a section of the journal

Frontiers in Oncology

Received: 01 March 2021

Accepted: 11 May 2021

Published: 26 May 2021

Citation:

Nakamura $Y$, Takada M, Imamura $M$,

Higami A, Jiaxi $H$, Fujino $M$,

Nakagawa $R$, Inagaki $Y$, Matsumoto $Y$,

Kawaguchi K, Kawashima M, Suzuki E

and Toi M (2021) Usefulness and

Prospects of Sentinel Lymph Node

Biopsy for Patients With Breast Cancer

Using the Medical Imaging

Projection System.

Front. Oncol. 11:674419.

doi: 10.3389/fonc.2021.674419

\section{Usefulness and Prospects of Sentinel Lymph Node Biopsy for Patients With Breast Cancer Using the Medical Imaging Projection System}

Yuki Nakamura ${ }^{1}$, Masahiro Takada ${ }^{1 *}$, Michiko Imamura $^{2}$, Akane Higami $^{1}, \mathrm{He}$ Jiaxi ${ }^{1}$, Makoto Fujino ${ }^{1}$, Rie Nakagawa ${ }^{1}$, Yukiko Inagaki ${ }^{1}$, Yoshiaki Matsumoto ${ }^{1}$, Kosuke Kawaguchi ${ }^{1}$, Masahiro Kawashima ${ }^{1}$, Eiji Suzuki ${ }^{1}$ and Masakazu Toi ${ }^{1}$

${ }^{1}$ Department of Surgery (Breast Surgery), Kyoto University Hospital, Kyoto, Japan, ${ }^{2}$ Department of Breast and Endocrine
Surgery, Hyogo College of Medicine, Hyogo, Japan

Background: The Medical Imaging Projection System (MIPS) projects indocyanine green (ICG) fluorescence images directly on the surgical field using a projection mapping technique. We conducted an observational study of sentinel lymph node (SLN) biopsy using the prototype MIPS; we found a high identification rate. However, the number of SLN-positive cases was small, and the sensitivity could not be evaluated. The aim of this study was to investigate the clinical usefulness of the MIPS assisted ICG fluorescence method using commercially available equipment.

Methods: This was a retrospective observational study. Patients with primary breast cancer who underwent SLN biopsy using the MIPS at Kyoto University Hospital from April to December 2020 were included in the study. The primary endpoints were the identification rate of SLNs and detection of positive SLNs by the MIPS. The secondary endpoint was the number of SLNs excised using the MIPS per patient. We also conducted a questionnaire survey focused on the utility of the MIPS; it involved doctors with an experience in using the MIPS.

Results: Seventy-nine patients (84 procedures) were included in the study. In 60 (71\%) procedures, both the radioisotope (RI) method and MIPS were used. At least one SLN could be detected by the MIPS in all the procedures, with an identification rate of $100 \%$ (95\% confidence interval 95.6-100\%). A total of 19 (7\%) positive SLNs were removed, which were identifiable by the MIPS. Among 57 patients in whom the MIPS and RI methods were used, there was no positive SLN only identified by the RI method. The results of the questionnaire survey showed that the MIPS enabled the operator and assistant to share the ICG fluorescence image in the surgical field and to communicate with each other easily. 
Conclusion: The current study demonstrated that the identification rate of SLNs using the MIPS was high, and the MIPS can be used for detecting positive SLNs. It was suggested that the MIPS will be useful in learning SLN biopsy procedures.

Keywords: breast cancer, sentinel lymph node biopsy, indocyanine green fluorescence method, projection mapping, identification rate

\section{INTRODUCTION}

Sentinel lymph node (SLN) biopsy is the standard of care for patients with clinically node-negative breast cancer. In addition to the radioisotope (RI) and blue dye methods, SLN identification with indocyanine green (ICG) fluorescence was first reported in 2005 (1). From a prior meta-analysis, the SLN identification rates using the ICG fluorescence method and RI method were similar (2).

The Medical Imaging Projection System (MIPS) is a novel near infrared (NIR) fluorescence imaging system that projects ICG fluorescence images directly on the surgical field using a projection mapping technique. The MIPS assisted ICG fluorescence method involves an accurate and continuous projection of ICG fluorescence signals, which enables a realtime navigation surgery for SLN biopsy without shifting the visual focus from the surgical field. It does not require operating lights. In 2018, we conducted an observational study of SLN biopsy using the prototype MIPS in 56 patients (59 procedures) at our institution; the identification rate was comparable to that obtained with the ICG fluorescence method using a conventional NIR system. However, the low percentage of patients with SLN metastasis led to insufficient evaluation of detection of positive SLNs by the MIPS (3).

The aim of this study was to assess SLN identification and detection of positive SLNs using the commercial-type MIPS. We also conducted a questionnaire survey on the utility of the MIPS.

\section{PATIENTS AND METHODS}

\section{Patients}

The eligibility criteria included: histologically confirmed breast cancer, clinically node-negative tumor, and patients who underwent SLN biopsy using the MIPS. Patients with a previous history of axillary surgery were excluded. Patients who received preoperative systemic therapy (PST) were included.

The study protocol was approved by the institutional review board of Kyoto University Hospital.

\section{Methods}

This was a retrospective observational study. Data on age, body mass index (BMI), tumor size, tumor histology, subtype, with or without PST, number of SLNs, and combined use of the RI method were extracted from the medical records. We also conducted a questionnaire survey involving doctors at Kyoto University Hospital and the Hospital of Hyogo College of Medicine with experience in using the MIPS.
Detailed features of MIPS and its surgical procedures have been reported previously (4). The commercial-type MIPS was used in this study, in which flexibility of the projection head was improved by introducing the same arm control technology as the surgical microscope; surgeons were able to adjust the projection angle freely during the procedures (Figures 1 and 2).

\section{Endpoints}

The primary endpoints included the identification rate of SLNs and detection of positive SLNs by the MIPS. The identification rate was calculated as the percentage of all patients in whom at least one SLN was detected using the MIPS. The detection of positive SLNs was evaluated using the percentage of positive SLNs among all the excised SLNs.

The secondary endpoints included: the number of SLNs excised by the MIPS per patient, relationship between clinical factors and number of detected SLNs, and the detection of SLNs by the MIPS and the RI method among patients after PST. All statistical analyses were performed using JMP ${ }^{\circledR}$ Pro (ver. 15.2.0; SAS Institute, Inc. Cary, NC, USA).

\section{RESULTS}

A total of 79 patients (74 and 5 with unilateral and bilateral tumors, respectively) underwent SLN biopsy using the MIPS from April to December 2020. The analysis was based on 84 procedures. The median age was 59 (range 35-84) years, and the median BMI was 21.1 (range 16.4-32.6) $\mathrm{kg} / \mathrm{m}^{2}$. More than half of the procedures were performed for $\mathrm{T} 1$ tumors (55\%), and 15 (18\%) procedures were performed for ductal carcinomas in situ. Of the 15 patients with DCIS, 13 patients underwent mastectomy and 2 patients were suspected of having invasive carcinoma by imaging findings. Of the 84 procedures, 11 (13\%) were performed after PST and all of these patients were clinical node negative. The RI and MIPS methods were both used in $60(71 \%)$ procedures of 57 patients (Table 1).

The identification rate of the MIPS was 100\% (84/84: 95\% confidence interval [CI] 95.6-100\%). Macrometastases were found in 19 (7\%) of the 256 SLNs, detected by the MIPS.

Of 185 SLNs excised among the patients who underwent SLN biopsy using both the MIPS and RI methods, 90 (49\%) SLNs were identified by both the MIPS and RI methods, 91 (49\%) only by the MIPS, 2 (1\%) only by the RI method, and $2(1 \%)$ by neither the MIPS nor RI method. The number of positive SLNs detected by both the MIPS and RI methods was 8 , that only by the MIPS was 1, that only by the RI method and by neither the MIPS nor RI method were 0 (Table 2). 


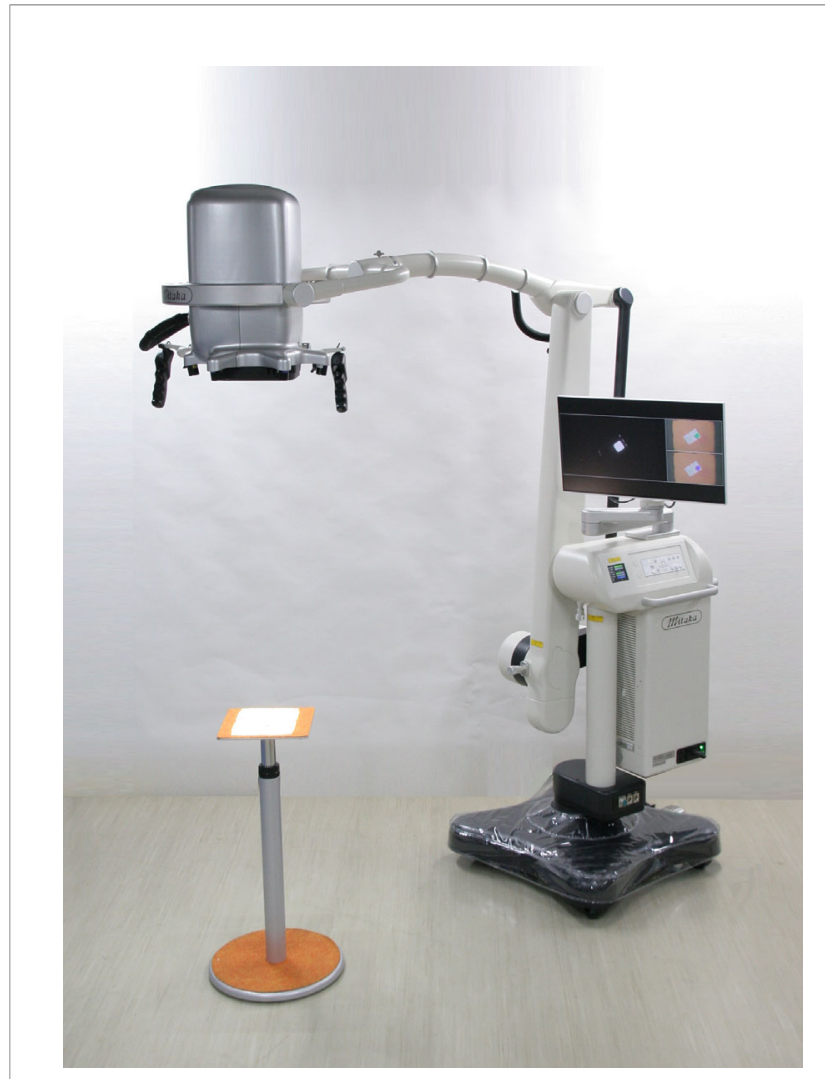

FIGURE 1 | The Medical Imaging Projection System (MIPS). Photograph of the commercial-type MIPS used in this study.

The median number of SLNs detected by the MIPS per patient was 3 (range, 1-6). The median number of SLNs identified by the MIPS among patients with a high BMI $\left(\geq 22 \mathrm{~kg} / \mathrm{m}^{2}\right)$ was almost equal to that of patients with a low BMI $\left(<22 \mathrm{~kg} / \mathrm{m}^{2}\right)(P=0.09)$. Of the procedures performed after PST, the median number of SLNs identified by the MIPS was 3 (range, 2-5) (Table 3 ).

Among patients who underwent SLN biopsy after PST, the identification rate of the MIPS was $100 \%$ (95\% CI 74.1-100\%) and that of the RI method was $100 \%$ (95\% CI 70.1-100\%). The median number of SLNs identified by the RI method was 1 (range, 1-3). The number of positive SLNs was one, which was detected by both the MIPS and RI methods.

The results of the questionnaire survey are shown in Table 4. Regarding the clinical advantage of the MIPS, $69 \%$ of the doctors answered that operators could perform the procedures easily by confirming the ICG fluorescence image directly in the surgical field; $54 \%$ answered that operators could perform the procedures without disrupting the surgical workflow since the MIPS did not require operating lights during the procedures; $62 \%$ answered that operators could operate smoothly without holding the NIR camera, and $62 \%$ answered that both the operator and assistant could communicate with each other easily by sharing the ICG fluorescence image in the surgical field during the surgery. Regarding the relationship between the MIPS and procedure difficulty including duration of surgery, $38 \%$ of the doctors felt
TABLE 1 | Patient and tumor characteristics.

\begin{tabular}{|c|c|c|}
\hline Factors & $\mathbf{N}$ & $\%$ \\
\hline All procedures & 84 & \\
\hline Age, median (range) & $59(35-84)$ & \\
\hline Body Mass Index, median (range) & $21.1(16.4-32.6)$ & \\
\hline \multicolumn{3}{|l|}{ Tumor stage } \\
\hline Tis & 15 & 18 \\
\hline $\mathrm{T} 1 \mathrm{a}$ & 6 & 7 \\
\hline $\mathrm{T} 1 \mathrm{~b}$ & 10 & 12 \\
\hline $\mathrm{T} 1 \mathrm{c}$ & 30 & 36 \\
\hline $\mathrm{T} 2$ & 21 & 25 \\
\hline T3 & 1 & 1 \\
\hline \multicolumn{3}{|l|}{ Histology } \\
\hline Ductal Carcinoma in situ & 15 & 18 \\
\hline Invasive carcinoma (no special type) & 60 & 71 \\
\hline Invasive lobular carcinoma & 5 & 6 \\
\hline Others & 4 & 5 \\
\hline \multicolumn{3}{|l|}{ Subtype } \\
\hline Hormone-receptor-positive and HER2-negative & 53 & 63 \\
\hline Hormone-receptor-positive and HER2-positive & 6 & 7 \\
\hline Hormone-receptor-negative and HER2-positive & 2 & 2 \\
\hline Triple negative & 8 & 10 \\
\hline Unknown & 15 & 18 \\
\hline \multicolumn{3}{|l|}{ Histological grade } \\
\hline 1 & 16 & 19 \\
\hline 2 & 30 & 36 \\
\hline 3 & 23 & 27 \\
\hline Unknown & 15 & 18 \\
\hline \multicolumn{3}{|l|}{ Preoperative systemic therapy } \\
\hline Yes & 11 & 13 \\
\hline No & 73 & 87 \\
\hline \multicolumn{3}{|l|}{ Combined use of radioisotope method } \\
\hline Yes & 60 & 71 \\
\hline No & 24 & 29 \\
\hline
\end{tabular}

HER2, human epidermal growth factor receptor 2.

that the MIPS led to a shorter duration of surgery, 31\% did not feel so, and $31 \%$ were indifferent.

\section{DISCUSSION}

The current study showed the reproducibility of the MIPS for SLN identification and the feasibility of detection of positive SLNs by the MIPS.

It has been reported that the SLN identification rate ranged from $89-100 \%$, and the mean number of removed SLNs was 1.53.4, using the ICG fluorescence method (2). In the current study, the SLN identification rate was 100\% (95\% CI: 95.6-100\%), and the median number of SLNs detected by MIPS was 3 (range 1-6), and our results were comparable to those of the ICG fluorescence method using a conventional NIR system.

In this study, $17 \%$ of the patients had positive SLNs, which was higher than that in our previous study; however, it was lower than those in previous reports (5). Preoperative examination of axillary lymph nodes in our institution includes palpation, ultrasonography, and contrast-enhanced magnetic resonance imaging (CE-MRI). If nodal involvement is suspected, ultrasound-guided fine-needle aspiration biopsy (US-FNA) is also performed. CE-MRI and US-FNA increase the accuracy of 
A

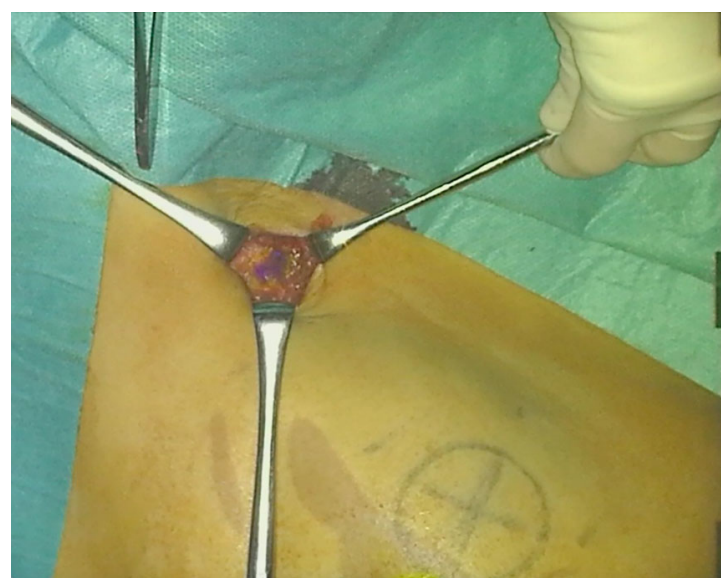

B

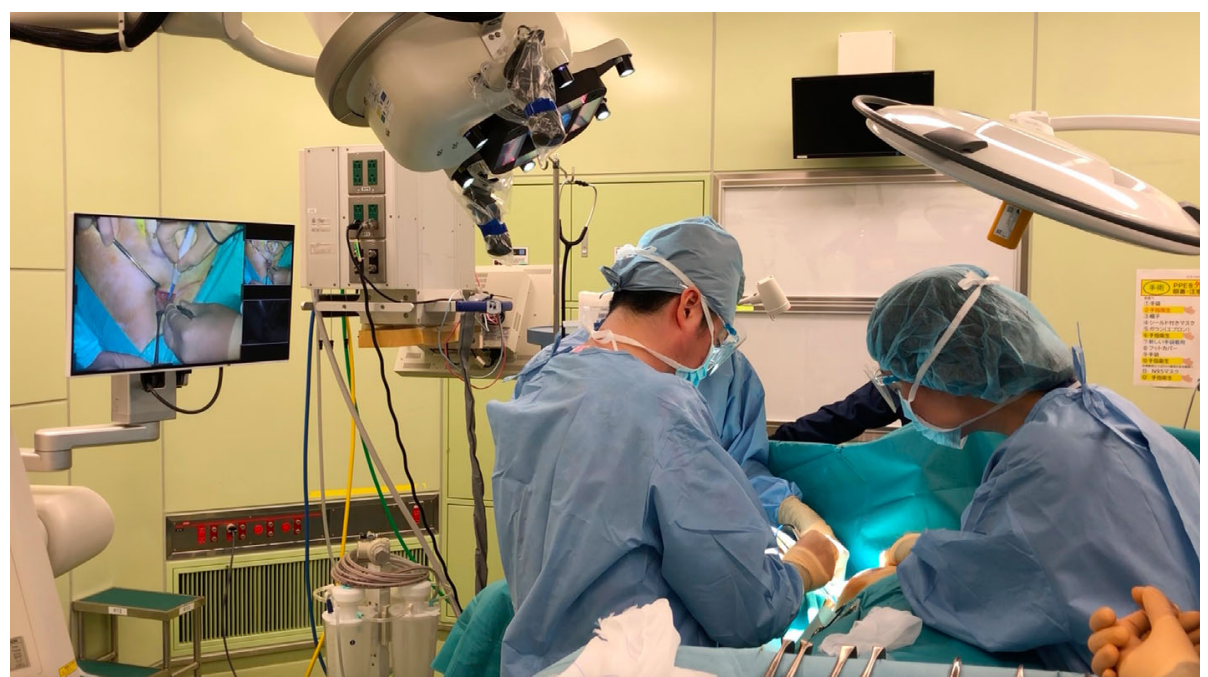

FIGURE 2 | Intraoperative photograph of using the MIPS. (A) Operator can confirm the ICG fluorescence image directly in the surgical field. (B) Both the operator and assistant can share the ICG fluorescence image in the surgical field.

TABLE 2 | The number of SLNs among cases using MIPS and RI

\begin{tabular}{lcc}
\hline SLN biopsy procedures & $\begin{array}{c}\text { Identified SLNs } \\
\text { (total } \mathrm{N}=185)\end{array}$ & $\begin{array}{c}\text { Positive SLNs } \\
\text { (total } \mathrm{N}=9 \text { ) }\end{array}$
\end{tabular}

MIPS and RI

MIPS only

RI only

Neither MIPS nor RI

90

91

91
2

2

(Palpation)

$S L N$, sentinel lymph node; MIPS, medical imaging projection system; $R$ l, radioisotope.

preoperative axillary staging $(4,6)$; the involvement of these examinations may have contributed to the low proportion of positive SLNs in our study. In this study, there was no positive SLN that was identified only by the RI method, and the MIPS
TABLE 3 | Number of SLNs identified by the MIPS according to patient characteristics.

\begin{tabular}{lcccc}
\hline Characteristics & N & Median & Range & P value \\
\hline $\begin{array}{l}\text { All procedures } \\
\text { BMl }\end{array}$ & 84 & 3 & $1-6$ & \\
$<22 \mathrm{~kg} / \mathrm{m}^{2}$ & 46 & 3 & $1-6$ & 0.09 \\
$\geqq 22 \mathrm{~kg} / \mathrm{m}^{2}$ & 38 & 2.5 & $1-5$ & \\
PST & & 3 & & 0.75 \\
Yes & 11 & 3 & $2-5$ & \\
No & 73 & & $1-6$ &
\end{tabular}

SLN, sentinel lymph node; BMI, body mass index; PST, preoperative systemic therapy.

identified all the positive SLNs in the patients in whom both the RI method and the MIPS were used. It is suggested that the detection of positive SLNs by the MIPS is comparable to detection by the RI method. 
TABLE 4 | Results of the questionnaire survey for doctors with an experience in using MIPS.

Which do you consider to be a clinical advantage of MIPS?

Operators can perform the procedures easily by confirming the ICG fluorescence image directly in the surgical field. Operators can perform the surgery without disruption of the surgical workflow since MIPS does not require operating lights. Operators can operate smoothly without holding the NIR camera.

The operator and assistant can communicate with each other easily due to the possibility of sharing the ICG fluorescence image. Do you feel the MIPS leads to shorter surgery duration?

Yes

No

Neither

$\begin{array}{ll}9 & 69 \\ 7 & 54 \\ 8 & 62 \\ 8 & 62 \\ & \\ 5 & 38 \\ 4 & 31 \\ 4 & 31\end{array}$

MIPS, medical imaging system projection system; ICG, indocyanine green.

It has been reported that the number of detected SLNs decreases as the BMI increases (7). However, in our study, the number of SLNs excised from patients with a high BMI was almost equal to that from patients with a low BMI. The MIPS may be useful in identifying SLNs among patients with a high BMI. We used BMI 22 as cutoff value, following the domestic standard value in Japan. There was no statistically significant difference in the number of sentinel lymph node identified, when we used the cutoff value of 25,28 and 30 (p value $0.33,0.75$ and 0.62 , respectively).

SLN biopsy after PST is feasible for patients with clinically node-negative cancer at baseline $(8,9)$. However, SLN biopsy after PST for patients with clinically node-positive cancer has a low identification rate and a high false-negative rate (10-12). Removal of three or more SLNs has been reported to reduce the false-negative rate to below $10 \%$ in patients with clinically nodepositive cancer (13). In the present study, SLN biopsies after PST were performed in $11(13 \%)$ procedures. Although this is a small number of cases, the identification rate by the MIPS among them was $100 \%$ (95\% CI $74.1-100 \%$ ), and the median number of excised SLNs was 3 (range 2-5).

The main clinical advantage of the MIPS is the ease of communication between the operator and assistant due to the possibility of sharing the ICG fluorescence image in the surgical field. From the results of the questionnaire survey, more than half of the doctors confirmed this advantage. The percentage of doctors who considered the communication between operator and assistant as a clinical advantage of the MIPS was 100\%,25\%, and $67 \%$ among doctors who graduated $\leq 5$ years, $6-10$ years, and $\geq 11$ years prior to the procedures. This tendency indicated that the communication between the operator and assistant would be useful in teaching the procedures to doctors willing to learn. We also expected a shorter duration of surgery with the MIPS, but the percentage of doctors who felt that using the MIPS shortened the duration of surgery was only $38 \%$.

There are several limitations to the present study. First, our sample size was relatively small; however, it did not hinder the clinical utility of the MIPS, as shown by the lower limit of the $95 \%$ CIs, which was above $90 \%$. Second, the retrospective nature of the study; however, our results were comparable to those of our previous prospective observational study. Third, the small number of SLN biopsies after PST; we need to investigate the utility of the MIPS in more cases after PST. Forth, the evaluation of the MIPS in learning procedures was not objective; we need to evaluate the learning curve of SLN biopsy procedures using the MIPS.

In conclusion, this study demonstrated that the identification rate of SLNs using the MIPS was high and the MIPS can be used for the detection of positive SLNs. The MIPS enabled an ease of communication between the operator and assistant. It was suggested that this advantage of the MIPS will be useful in learning SLN biopsy procedures.

\section{DATA AVAILABILITY STATEMENT}

The original contributions presented in the study are included in the article/supplementary material. Further inquiries can be directed to the corresponding author.

\section{ETHICS STATEMENT}

The studies involving human participants were reviewed and approved by Kyoto University Graduate School and Faculty of Medicine, Ethics Committee. Written informed consent for participation was not required for this study in accordance with the national legislation and the institutional requirements.

\section{AUTHOR CONTRIBUTIONS}

YN contributed to data collection and analysis and wrote this manuscript. MTa made important corrections to the manuscript. MI, AH, HJ, MF, RN, YI, YM, KK, MK, ES, and MTo answered the questionnaire survey and checked the final version of the manuscript. All authors contributed to the article and approved the submitted version.

\section{ACKNOWLEDGMENTS}

The authors thank doctors of Department of Breast and Endocrine Surgery of Hyogo College of Medicine for answering the questionnaire survey. 


\section{REFERENCES}

1. Kitai T, Inomoto T, Miwa M, Shikayama T. Fluorescence Navigation With Indocyanine Green for Detecting Sentinel Lymph Nodes in Breast Cancer. Breast Cancer (2005) 12:211-5. doi: 10.2325/jbcs.12.211

2. Sugie T, Ikeda T, Kawaguchi A, Shimizu A, Toi M. Sentinel Lymph Node Biopsy Using Indocyanine Green Fluorescence in Early-Stage Breast Cancer: A MetaAnalysis. Int J Clin Oncol (2017) 22:11-7. doi: 10.1007/s10147-016-1064-z

3. Takada M, Takeuchi M, Suzuki E, Sato F, Matsumoto Y, Torii M, et al. RealTime Navigation System for Sentinel Lymph Node Biopsy in Breast Cancer Patients Using Projection Mapping With Indocyanine Green Fluorescence. Breast Cancer (2018) 25:650-5. doi: 10.1007/s12282-018-0868-2

4. Houssami N, Ciatto S, Turner RM, Cody HS III, Macaskill P. Preoperative Ultrasound-Guided Needle Biopsy of Axillary Nodes in Invasive Breast Cancer: Meta-Analysis of its Accuracy and Utility in Staging the Axilla. Ann Surg (2011) 254:243-51. doi: 10.1097/SLA.0b013e31821f1564

5. Krag DN, Anderson SJ, Julian TB, Brown AM, Harlow SP, Ashikaga T, et al. Technical Outcomes of Sentinel-Lymph-Node Resection and Conventional Axillary-Lymph-Node Dissection in Patients With Clinically Node-Negative Breast Cancer: Results From the NSABP B-32 Randomised Phase III Trial. Lancet Oncol (2007) 8:881-8. doi: 10.1016/S1470-2045(10)70207-2

6. Liang X, Yu J, Wen B, Xie J, Cai Q, Yang Q. MRI and FDG-PET/CT Based Assessment of Axillary Lymph Node Metastasis in Early Breast Cancer: A MetaAnalysis. Clin Radiol (2017) 72:295-301. doi: 10.1016/j.crad.2016.12.001

7. Goyal A, Newcombe RG, Chhabra A, Mansel REALMANAC Trialists Group. Group at. Factors Affecting Failed Localisation and False-Negative Rates of Sentinel Node Biopsy in Breast Cancer-Results of the ALMANAC Validation Phase. Breast Cancer Res Treat (2006) 99:203-8. doi: 10.1007/ s10549-006-9192-1

8. Classe JM, Bordes V, Campion L, Mignotte H, Dravet F, Leveque J, et al. Sentinel Lymph Node Biopsy After Neoadjuvant Chemotherapy for Advanced Breast Cancer: Results of Ganglion Sentinelle Et Chimiotherapie Neoadjuvante, a French Prospective Multicentric Study. J Clin Oncol (2009) 27:726-32. doi: 10.1200/JCO.2008.18.3228
9. van Deurzen $\mathrm{CH}$, Vriens BE, Tjan-Heijnen VC, van der Wall E, Albregts M, van Hilligersberg R, et al. Accuracy of Sentinel Node Biopsy After Neoadjuvant Chemotherapy in Breast Cancer Patients: A Systematic Review. Eur J Cancer (2009) 45:3124-30. doi: 10.1016/j.ejca.2009.08.001

10. Boughey JC, Suman VJ, Mittendorf EA, Aherndt GM, Wilke LG, Taback B, et al. Sentinel Lymph Node Surgery After Neoadjuvant Chemotherapy in Breast Cancer Patients: A Systematic Review. Eur J Cancer (2009) 45:3124-30. doi: 10.1016/j.ejca.2009.08.001

11. Boileau JF, Poirier B, Basik M, Holloway CM, Gaboury L, Sideris L, et al. Sentinel Node Biopsy After Neoadjuvant Chemotherapy in Biopsy-Proven Node-Positive Breast Cancer: The SN FNAC Study. J Clin Oncol (2015) 33:258-64. doi: 10.1200/JCO.2014.55.7827

12. Kuehn T, Bauerfeind I, Fehm T, Fleige B, Hausschild M, Helms G, et al. Sentinel-Lymph-Node Biopsy in Patients With Breast Cancer Before and After Neoadjuvant Chemotherapy (SENTINA): A Prospective, Multicentre Cohort Study. Lancet Oncol (2013) 14:609-18. doi: 10.1016/S1470-2045(13) 70166-9

13. El Hage Chehade H, Headon H, El Tokhy O, Heeney J, Kasem A, Mokbel K. Is Sentinel Lymph Node Biopsy a Viable Alternative to Complete Axillary Dissection Following Neoadjuvant Chemotherapy in Women With NodePositive Breast Cancer at Diagnosis? Updated Meta-Analysis Involving 3,398 Patients. Am J Surg (2016) 212:969-81. doi: 10.1016/j.amjsurg.2016.07.018

Conflict of Interest: The authors declare that the research was conducted in the absence of any commercial or financial relationships that could be construed as a potential conflict of interest.

Copyright (c) 2021 Nakamura, Takada, Imamura, Higami, Jiaxi, Fujino, Nakagawa, Inagaki, Matsumoto, Kawaguchi, Kawashima, Suzuki and Toi. This is an open-access article distributed under the terms of the Creative Commons Attribution License (CC BY). The use, distribution or reproduction in other forums is permitted, provided the original author(s) and the copyright owner(s) are credited and that the original publication in this journal is cited, in accordance with accepted academic practice. No use, distribution or reproduction is permitted which does not comply with these terms. 